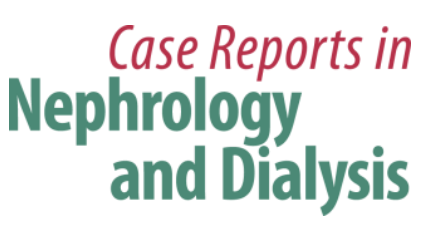

Case Rep Nephrol Dial 2020;10:65-70

DOI: $10.1159 / 000508126$

Published online: June 12, 2020

(C) 2020 The Author(s)

Published by S. Karger AG, Basel

www.karger.com/cnd

This article is licensed under the Creative Commons Attribution-NonCommercial 4.0 International License (CC BY-NC) (http://www.karger.com/Services/OpenAccessLicense). Usage and distribution for commercial purposes requires written permission.

\title{
Peritoneal Dialysis Zoonotic Bacterial Peritonitis with Staphylococcus pseudintermedius
}

\author{
Layth Dahbour $^{\mathrm{a}} \quad$ Jeffrey Gibbs ${ }^{\mathrm{a}} \quad$ Christian Coletta $^{\mathrm{a}} \quad$ Jeannine Hummell ${ }^{\mathrm{b}}$ \\ Mohammad Al-Sarie $^{a} \quad$ Navkirat Pannu Kahlona ${ }^{a}$ Basil Akpunonu ${ }^{a}$ \\ ${ }^{a} G$ eneral Internal Medicine, University of Toledo Medical Center, Toledo, OH, USA; \\ bepartment of Nursing, ProMedica Toledo Hospital, Toledo, OH, USA
}

\section{Keywords}

Dialysis · Peritoneal dialysis · Peritonitis · Staphylococcus pseudintermedius · Zoonotic bacteria

\begin{abstract}
We present the first reported case of peritoneal dialysis-associated peritonitis caused by Staphylococcus pseudintermedius, an organism that had been misclassified as S. aureus in the past. S. pseudintermedius is well recognized in the veterinary literature and noted as flora in the mouth, nares, and anus of domesticated animals. It has been associated with soft tissue infections in pets and is now being reported in increased frequency as the causative agent in various human infections. It also has a different antibiotic sensitivity profile. The patient had close contact with her pet dog and was successfully treated with intravenous antibiotics in the hospital followed by oral doxycycline for 10 days after discharge. The patient has not had any recurrent infection after obtaining and applying appropriate hygienic education and precautions.
\end{abstract}

\section{Introduction}

Peritonitis remains the most common complication of peritoneal dialysis (PD). It contributes to approximately $16 \%$ of all deaths in PD patients [1]. 


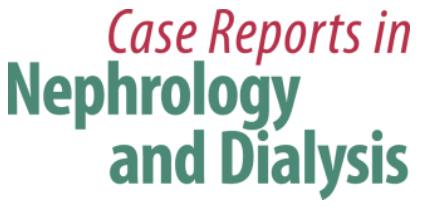

Case Rep Nephrol Dial 2020;10:65-70

DOI: $10.1159 / 000508126$

(c) 2020 The Author(s). Published by S. Karger AG, Basel www.karger.com/cnd

Dahbour et al.: PD Zoonotic Bacterial Peritonitis with Staphylococcus pseudintermedius

Common etiological agents of PD-associated peritonitis include coagulase-negative Staphylococcus, S. aureus, Pseudomonas aeruginosa, and other gram-negative organisms. Cases of polymicrobial, fungal, and non-tuberculosis mycobacterium PD-associated peritonitis have been documented as well [2-5]. However, it is important to consider uncommon causative organisms that are now being recognized and have been aided by various emerging genetic approaches that speciate infective organisms by their nucleic acid sequencings. Therefore, obtaining a comprehensive history from patients, timely peritoneal fluid analysis, and accurate identification of infecting organisms with current technology and treatments guided by an appropriate antimicrobial sensitivity pattern are important in current clinical practice to protect PD patients from misdiagnosis and potential treatment failures.

S. pseudintermedius is one of 38 species and 17 subspecies of the Staphylococcus genus and has been cultured from the oral cavity, anus, and nares of domesticated animals. In the last decade, the bacterium has been implicated in many soft tissue infections in domesticated dogs and to a lesser extent in cats. A study by Rubin and Chirino-Trejo [6] reports that $S$. pseudintermedius is the leading cause of pyoderma, otitis externa, and the most commonly isolated pathogen in urinary tract infections in companion dogs. It is a coagulase-positive Staphylococcus that exhibits many similar characteristics of the aureus species, but recent technological advances have provided evidence for its misclassification in the past [7].

There have been few published reports of S. pseudintermedius infections in humans and showing a strong correlation between the infection and animal companionship status [8]. The pathogenesis and site of infection have varied among patients with most involving the skin and soft tissue. We report the first published case of PD-associated bacterial peritonitis caused by S. pseudintermedius with close contact to a pet dog in a PD patient constituting a zoonotic infection. We also believe, based on this report, that extensive history taking that includes pet ownership is necessary for diagnosis of such unusual bacteria that can cause such human infections as peritonitis in PD patients.

\section{Case Presentation}

A 39-year-old female presented to the emergency department with complaints of epigastric pain of 2 days duration. Pain was described as gradual in onset, crampy, 10/10 severity scale with no exacerbating or relieving factors. The patient has a history of chronic kidney disease (CKD 5) from hypertension and was recently started on continuous ambulatory PD through a peritoneal catheter placed 8 weeks prior to admission. The patient denied chills or fevers on presentation. Physical examination revealed elevated blood pressure at 192/122 $\mathrm{mm} \mathrm{Hg}$, oral temperature $37.2^{\circ} \mathrm{C}$, pulse rate 78 beats per minute, and respiratory rate 18 breaths per minute. Abdominal examination revealed diffuse tenderness with no guarding or rebound tenderness. The PD catheter site showed mild redness but without any pus drainage or pus pockets on palpation of surrounding areas.

Complete blood count showed leukocytosis of $13,100 \times 10^{6}$ cell/L (80.8\% neutrophils, $12.9 \%$ lymphocytes, $4.3 \%$ monocytes, $1.6 \%$ eosinophils, and $0.4 \%$ basophils). A computed tomographic image of the abdomen and pelvis did not reveal any inflammatory process. Peritoneal fluid analysis from the exit site of the peritoneal catheter showed 2,505 nucleated cells/ $\mu \mathrm{L}$ with $92 \%$ neutrophils and $8 \%$ lymphocytes, and no organism identified on gram stain. A presumptive diagnosis of bacterial peritonitis was made, and the patient was started on intravenous vancomycin and piperacillin-tazobactam for empiric coverage pending culture results. By the second day of admission, the patient's abdominal pain improved, and a repeat 


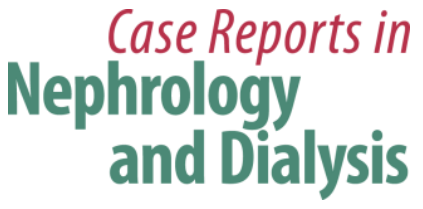

Case Rep Nephrol Dial 2020;10:65-70

DOI: $10.1159 / 000508126$

(c) 2020 The Author(s). Published by S. Karger AG, Basel www.karger.com/cnd

Dahbour et al.: PD Zoonotic Bacterial Peritonitis with Staphylococcus pseudintermedius

PD fluid analysis showed a $90 \%$ reduction of nucleated cells to 61 cells $/ \mu \mathrm{L}$. The peritoneal dialysate grew cultures of a gram-positive coccus, later identified as S. pseudintermedius, which was not methicillin resistant.

Upon further interview, the patient reported ownership and closeness to her Pitt bull dog. The dog sleeps with the patient and is in close contact with various portions of the PD component. She was not careful that her dog should be kept away from her PD catheter to prevent infections. We suspected that her dog's close proximity to her PD catheter site was the source of $S$. pseudintermedius infection. The patient responded well to intravenous antibiotics and was later discharged home on a 10-day course of oral doxycycline $100 \mathrm{mg}$ twice daily as the sensitivity pattern did not show any resistance to tetracycline. No recurrent infections have been noted since admission with better hygiene and some distance with the pet dog.

\section{Discussion}

S. pseudintermedius is a coagulase-positive coccus organism that shares various phenotypic morphologies with the aureus species. Both are catalase positive, coagulase positive, form ß-hemolytic colonies on sheep agar, ferment mannitol on mannitol salt agar, and exhibit positive DNase activities on agar plates. The shared characteristics between the organisms can lead to false and mis-identification of S. pseudintermedius as $S$. aureus in human infections with potential for treatment failures. While biochemical tests as pyrrolidonyl arylamidase are positive for S. pseudintermedius and negative for S. aureus, the employment of recent technologies have provided additional methodologies to distinguish between them. The utilization of VITEK-2 technology with its multichannel fluorimetry and photometric signals are now used in the rapid and accurate identification of various organisms including staphylococcal species and S. pseudintermedius. In addition, the application of matrix-assisted laser desorption ionization time of flight mass spectrometry (MALDI TOF MS) is being used increasingly to speciate more accurately common bacteria including staphylococcal species [7, 8]. This technology generates ribosomal protein fingerprints of an organism that is then compared to a database to assign an identity to the isolate as in our case. As a result, S. pseudintermedius is now being recognized as a cause of human infections with increasing frequency [9].

Since morbidity and mortality associated with PD peritonitis is high, a thorough history before and after identification of organisms helps direct physicians to more accurate care with antibiotic choices, promotion of better prevention, and infection control protocols.

In this reported case, the peritoneal dialysate fluid grew S. pseudintermedius, prompting search for possible source of the bacteria. While most human infections with S. pseudintermedius are in the skin and soft tissues, rare cases of prosthetic and bloodstream infections are being reported $[9,10]$.

Peritonitis with S. pseudintermedius in peritoneal dialysate has not been reported previously in humans, and the fact that the organism was cultured from a pet owner on PD who is not practicing adequate hygienic protocols suggest an animal-to-human transmission. It was not feasible at the time to obtain cultures from the pet dog, but the epidemiology and what is known about the bacteria lead us to believe that the infection came from her companion dog.

With high pet ownership in most populations, the importance of zoonotic spread merits closer observation [11,12]. Dog ownership was found statistically significant in predicting $S$. pseudintermedius human colonization and infections $[9,12,13]$. The spectrum of infections caused by $S$. pseudintermedius resembles that of $S$. aureus in humans. Methicillin-resistant $S$. pseudintermedius (MRSP) is also being reported in companion animals. It is also reported that 


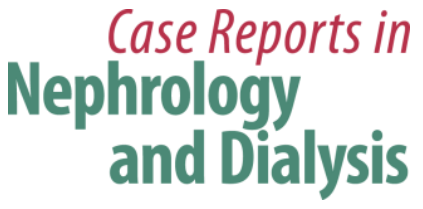

Case Rep Nephrol Dial 2020;10:65-70

DOI: $10.1159 / 000508126$

(c) 2020 The Author(s). Published by S. Karger AG, Basel www.karger.com/cnd

Dahbour et al.: PD Zoonotic Bacterial Peritonitis with Staphylococcus pseudintermedius

since 2006, MRSP has become a significant health problem for companion cats and dogs [11, $14,15]$. A proposed mechanism for emerging antimicrobial resistance includes the transfer of SCCmec genes between different staphylococcal species [16]. Although incidences of MRSP infections have been rare in humans, the potential transfer of antimicrobial resistant genes could eventually harm humans in similar patterns as methicillin-resistant $S$. aureus.

MRSP are commonly resistant to doxycycline and trimethoprim/sulfamethoxazole, such resistance to the two agents is uncommon with methicillin-resistant $S$. aureus (MRSA), hence accurate diagnosis is crucial [15]. The bacterial sensitivity pattern in this patient did not reveal any resistance to tetracycline, and patient was discharged on oral doxycycline.

The variability among dialysis training programs may leave some patients without adequate and complete competence in the prevention of catheter-related infections [17]. Adequate dialysis training is therefore critical to decrease the risk of PD-associated infections and peritonitis with emphasis on the prevention of peritonitis with an adequate hygiene protocol and surveillance. Such education can decrease the $16 \%$ mortality in PD patients. A history of pet ownership should be an integral part of such educational process especially in those with pet companions, since the infective organisms may differ.

\section{Conclusions}

Pet ownership puts PD patients at an increased risk of developing peritonitis due to pathogens common in dogs or cats when routine hygiene techniques are not adequately employed. S. pseudintermedius is one such common bacterium in companion animals that is reported on increased frequency in the veterinary medicine literature and now causing various human infections. We report the first case of PD-associated peritonitis caused by S. pseudintermedius and suggest that accurate diagnosis is imperative as its microbiology is different and could pose harm to humans. Furthermore, PD training and ongoing education programs are needed for pet owners to help decrease the risk of PD-associated bacterial peritonitis.

\section{Acknowledgement}

Thank you to Brenda Joyce for her administrative support and assistance with the submission of this case report.

\section{Statement of Ethics}

The research was conducted ethically in accordance with the World Medical Association Declaration of Helsinki.

A written informed consent for the publication of this case report was obtained from the patient by the senior author B.A. with stipulation that her identifiable attributes must be anonymized in the manuscript.

The University of Toledo Biomedical IRB does not require IRB review of case reports that have 3 or fewer subjects. The basis for this is that Federal Regulations 45 CFR 46 requires IRB review only when human studies are generalizable, which is not the case for small case reports. Thus, this case report of a single subject does not require IRB review. We also noted on review of the report that there is no HIPAA protected medical information included. 


\section{Case Reports in Nephrology and Dialysis}

\section{Disclosure Statement}

The authors have nothing to disclose.

\section{Funding Sources}

There are no sources of funding to disclose.

\section{Author Contributions}

Jeffrey Gibbs, Christian Coletta, and Jeannine Hummell performed the literature search, data collection, data analysis, and wrote the first draft of the manuscript. Mohammad Al-Sarie and Navkirat Pannu Kahlon participated in subsequent revisions and evaluated the new genetic and molecular techniques for recent taxonomies. Layth Dahbour and Basil Akpunonu provided supervision and mentorship in the final revisions to the editor. All authors read and approved the final manuscript.

\section{References}

1 Akoh JA. Peritoneal dialysis associated infections: an update on diagnosis and management. World J Nephrol. 2012 Aug;1(4):106-22.

2 Zelenitsky SA, Howarth J, Lagacé-Wiens P, Sathianathan C, Ariano R, Davis C, et al. Microbiological Trends and Antimicrobial Resistance in Peritoneal Dialysis-Related Peritonitis, 2005 to 2014. Perit Dial Int. 2017 Mar-Apr;37(2):170-6.

3 Predari SC, de Paulis AN, Verón D, Zucchini A, Santoianni JE. Fungal peritonitis in patients on peritoneal dialysis: twenty five years of experience in a teaching hospital in Argentina. Rev Argent Microbiol. 2007 OctDec;39(4):213-7.

4 Barraclough K, Hawley CM, McDonald SP, Brown FG, Rosman JB, Wiggins KJ, et al. Polymicrobial peritonitis in peritoneal dialysis patients in Australia: predictors, treatment, and outcomes. Am J Kidney Dis. 2010 Jan;55(1):121-31.

5 Renaud CJ, Subramanian S, Tambyah PA, Lee EJ. The clinical course of rapidly growing nontuberculous mycobacterial peritoneal dialysis infections in Asians: A case series and literature review. Nephrology (Carlton). 2011 Feb;16(2):174-9.

6 Rubin JE, Chirino-Trejo M. Prevalence, sites of colonization, and antimicrobial resistance among Staphylococcus pseudintermedius isolated from healthy dogs in Saskatoon, Canada. J Vet Diagn Invest. 2011 Mar;23(2):351-4.

7 Börjesson S, Gómez-Sanz E, Ekström K, Torres C, Grönlund U. Staphylococcus pseudintermedius can be misdiagnosed as Staphylococcus aureus in humans with dog bite wounds. Eur J Clin Microbiol Infect Dis. 2015 Apr;34(4):839-44.

8 Murugaiyan J, Walther B, Stamm I, Abou-Elnaga Y, Brueggemann-Schwarze S, Vincze S, et al. Species differentiation within the Staphylococcus intermedius group using a refined MALDI-TOF MS database. Clin Microbiol Infect. 2014 Oct;20(10):1007-15.

9 Somayaji R, Priyantha MA, Rubin JE, Church D. Human infections due to Staphylococcus pseudintermedius, an emerging zoonosis of canine origin: report of 24 cases. Diagn Microbiol Infect Dis. 2016 Aug;85(4):471-6.

10 Starlander G, Börjesson S, Grönlund-Andersson U, Tellgren-Roth C, Melhus A. Cluster of infections caused by methicillin-resistant Staphylococcus pseudintermedius in humans in a tertiary hospital. J Clin Microbiol. 2014 Aug;52(8):3118-20.

11 Pomba C, Rantala M, Greko C, Baptiste KE, Catry B, van Duijkeren E, et al. Public health risk of antimicrobial resistance transfer from companion animals. J Antimicrob Chemother. 2017 Apr;72(4):957-68.

12 Broughton A, Verger C, Goffin E. Pets-related peritonitis in peritoneal dialysis: companion animals or trojan horses? Semin Dial. 2010 May-Jun;23(3):306-16.

13 Lozano C, Rezusta A, Ferrer I, Pérez-Laguna V, Zarazaga M, Ruiz-Ripa L, et al. Staphylococcus pseudintermedius Human Infection Cases in Spain: Dog-to-Human Transmission. Vector Borne Zoonotic Dis. 2017 Apr;17(4):268-70. 
14 Bannoehr J, Guardabassi L. Staphylococcus pseudintermedius in the dog: taxonomy, diagnostics, ecology, epidemiology and pathogenicity. Vet Dermatol. 2012 Aug;23(4):253-266, e251-252.

15 van Duijkeren E, Catry B, Greko C, Moreno MA, Pomba MC, Pyörälä S, et al.; Scientific Advisory Group on Antimicrobials (SAGAM). Review on methicillin-resistant Staphylococcus pseudintermedius. J Antimicrob Chemother. 2011 Dec;66(12):2705-14.

16 Ishihara K, Koizumi A, Saito M, Muramatsu Y, Tamura Y. Detection of methicillin-resistant Staphylococcus pseudintermedius ST169 and novel ST354 SCCmec II-III isolates related to the worldwide ST71 clone. Epidemiol Infect. 2016 Jan;144(2):434-42.

17 Piraino B, Bernardini J, Brown E, Figueiredo A, Johnson DW, Lye WC, et al. ISPD position statement on reducing the risks of peritoneal dialysis-related infections. Perit Dial Int. 2011 Nov-Dec;31(6):614-30. 